\title{
Study on the Enlargement Index of Femtosecond Laser-Assisted Capsulorhexis in 2-6-Year-Old Patients With Congenital Cataract
}

\section{Menglian Liao}

Central South University Aier School of Opthalmology https://orcid.org/0000-0002-5280-7557

\section{Da Guo}

Changsha Aier Eye Hospital

\section{Shan Liao}

Changsha Aier Eye Hospital

\section{Wenwen Zhang}

Central South University Aier School of Opthalmology

\section{Ding Lin}

Central South University Aier School of Opthalmology

Qiongyan Tang ( $\boldsymbol{\nabla}$ tangqiongyan@aierchina.com )

https://orcid.org/0000-0002-8148-0274

\section{Research article}

Keywords: anterior capsulorhexis enlargement index, capsulorhexis diameter, congenital cataract, femtosecond laser-assisted cataract surgery

Posted Date: August 7th, 2020

DOl: https://doi.org/10.21203/rs.3.rs-44987/v1

License: (9) This work is licensed under a Creative Commons Attribution 4.0 International License. Read Full License

Version of Record: A version of this preprint was published at BMC Ophthalmology on December 1st, 2021. See the published version at https://doi.org/10.1186/s12886-021-02184-y. 


\section{Abstract}

Background: To identify the capsule enlargement index after femtosecond laser-assisted anterior capsulorhexis in 2-6-year-old children who underwent congenital cataract surgery.

Methods: In this prospective case series study, femtosecond laser-assisted anterior capsulorhexis was performed in patients with congenital cataract, aged 2-6 years. The actual achieved capsulorhexis diameters were measured with Digimizer version 4.2.6. Correlation coefficient $(r)$ and multiple linear regression analysis were used to evaluate the variables that could potentially influence anterior capsulorhexis enlargement index (E).

Results: This prospective study enrolled 28 eyes of 22 patients with congenital cataract. The mean age of the patients at surgery was 4.67 years \pm 1.54 (standard deviation [SD]). " $E$ " of the 28 cases was $1.211 \pm 0.039(S D)$. Correlation analysis showed that " $E$ " correlated significantly with the anterior chamber depth (ACD) $(r=-0.469, p=0.021)$ and axial length $(A L)(r=0.452, p=0.027)$. The following formula was developed by using multivariable linear regression analysis: Predicted $E=1.177-0.052 \times A C D+0.009 \times A L$, $R^{2}=0.346(F=4.396, p=0.046)$.

Conclusions: The anterior capsulorhexis enlargement index and its calculation formula could help to set up an accurate programmed capsulorhexis diameter for femtosecond laser-assisted congenital cataract surgery in children aged 2-6 years. Thus, an appropriate actual capsulorhexis diameter could be achieved

\section{Background}

Congenital cataract is a relatively rare disease with an estimated prevalence of $2.2 / 10000-13.6 / 10000$ worldwide; ${ }^{1}$ however, it is the primary cause of childhood blindness. Congenital cataract accounts for $12 \%-39 \%$ of pediatric blindness cases in developing countries ${ }^{2}$. The period from birth till the age of 6 years is important for visual development in children ${ }^{3}$. Surgery should be performed during this period in patients with lens opacity, which leads to an unclear visual axis ${ }^{4}$. Continuous circular capsulorhexis (CCC) is the key and challenging step in cataract surgery, which influences the intraocular lens (IOL) position and postoperative refraction ${ }^{5-7}$.

Femtosecond laser-assisted cataract surgery (FLACS), which facilitates precise circularity and shape during cataract surgery, can make CCC safer and easier to achieve ${ }^{8,9}$. However, the presently achieved capsulorhexis size is definitely larger than that programmed for children. Therefore, to estimate the ideal CCC size, this study aimed to identify the laser capsulorhexis enlargement index and evaluate the factors affecting the index in 2-6-year-old children with congenital cataract. This work will help clinicians to set up an optimal programmed capsulorhexis diameter (PCD) and get accurate actual CCC size.

\section{Methods}


This prospective, consecutive case series study was conducted in patients with congenital cataract aged 2-6 years, who underwent FLACS between August 2017 and July 2019. All interventions were performed at the Changsha AIER Eye Hospital, AIER School of Ophthalmology, Central South University, Changsha, China. The study was approved by Changsha Aier Eye Hospital Review Board. Informed consent of the surgical video recording and publication of images and findings in this study was obtained from all patients' guardians and parents. The study protocol complied with the tenets of the Declaration of Helsinki.

The inclusion criteria were as follows: children with monocular or binocular congenital cataracts, femtosecond laser-assisted CCC with primary IOL implantation and posterior capsulotomy with anterior vitrectomy under general anesthesia, no history of ocular injury, no corneal pathology, no preoperative glaucoma, and no history of other surgeries. Patients with pupil diameter $<6 \mathrm{~mm}$ with full pharmacological mydriasis, intumescent white cataract and those with intraoperative capsule rupture were excluded.

\section{Surgical Technique}

FLACS was performed by a single experienced surgeon (QY Tang) in a sterile operating room. Using the femtosecond laser system (LenSx version 2.30; Alcon Laboratories, Inc., Fort Worth, Texas), an anterior capsulorhexis was programmed with $6 \mu \mathrm{J}$ energy and $300 \mu \mathrm{m}$ capsule delta up and down to create a 4.2-4.8 $\mathrm{mm}$ incision based on the surgeon's personal experience. A 2.2-mm corneoscleral limbus incision and a 1.0-mm side incision were created using sterile knives. After hydrodissection, nucleus removal and cortical aspiration were performed. Thereafter, an IOL was accurately implanted into the capsular bag. Viscoelastics present in the capsular bag and anterior chamber was completely sucked out. Then, a 3-4$\mathrm{mm}$ posterior capsulotomy was performed with anterior 23-gauge vitrectomy via the main incision and paracentesis. Finally, the main incision was hydrated and the paracentesis was closed with a 10-0 absorbable polyglycolic acid suture. The whole operation was recorded with a high-definition camera, and the recorded video was used for the following analysis.

\section{Video Review and Capsulorhexis Size Measurement}

Twenty-eight surgical videos of congenital cataract surgeries were reviewed. Images of the moment after suturing the incisions were captured and imported to a software for computer-assisted image analysis (Digimizer version 4.2.6, MedCalc Software Ltd, Mariakerke, Belgium). These images were used to measure the actual achieved capsulorhexis (Figure 1). Before performing the measurements, the radius of IOL optic is used to determine the unit. This measurement is crucial to assess the actual capsulorhexis. The detailed measurement procedure is shown in Figure 1. The ratio of the actual achieved capsulorhexis diameter (AACD) to the PCD was estimated as the capsule elasticity index (index of enlargement; E), which represents the degree of capsulorhexis enlargement.

\section{Clinical Data Collection}


Before the cataract surgery, patients underwent a thorough ophthalmologic examination, including a slit lamp evaluation, intraocular pressure measurement, ophthalmoscopy after mydriasis, as well as biometry and keratometry measurement. Axial length (AL) and anterior chamber depth (ACD) measurements were obtained from IOL-Master (Carl Zeiss Meditec, Jena, Germany) and A-scan ultrasonography (Cinescan $A / B$, Quantel Medical, France). The keratometry values (K1 and K2) were measured using autorefractor keratometer RK-F1 (Canon Co, Tokyo, Japan). The above ophthalmic parameters, as well as the age at cataract surgery, patient sex, and PCD were collected in this study.

\section{Statistical Analyses}

Simple correlation analysis and partial correlation analysis were used to evaluate the factors contributing to capsulorhexis enlargement. The data of normal distribution (age at surgery, K1 and K2) were evaluated by Pearson's correlation coefficient, while the non-normally distribution data (AL and ACD) were evaluated by Spearman's rank correlation coefficient. A multivariable linear regression model with equation was used to identify the various factors that influences capsulorhexis enlargement. All statistical analyses were performed using SPSS for Windows software (version 24.0, SPSS, Inc.), and a probability of $<5 \%$ $(p<0.05)$ was considered statistically significant.

\section{Results}

Twenty-eight eyes of 22 patients (20 [71.43\%] men and 8 [28.57\%] women) who underwent FLACS with primary IOL implantation were included in this study. The mean age at surgery was $4.67 \pm 1.54$ years. All measurements were performed preoperatively. Table 1 shows the characteristics of the eyes in the study population.

Table 1: Characteristics of patients' eyes.

\begin{tabular}{|llll|}
\hline Variables & Mean \pm SD & Median & Range \\
\hline Age at surgery $(\mathrm{y})$ & $4.67 \pm 1.54$ & 4.54 & $2.08,6.92$ \\
AL $(\mathrm{mm})$ & $22.33 \pm 1.71$ & 22.12 & $20.14,27.82$ \\
ACD $(\mathrm{mm})$ & $3.17 \pm 0.51$ & 3.33 & $1.57,3.93$ \\
K1 (D) & $42.36 \pm 2.08$ & 42.13 & $37.50,46.37$ \\
K2 (D) & $44.52 \pm 1.67$ & 44.31 & $41.98,48.21$ \\
PCD (mm) & $4.47 \pm 0.13$ & 4.40 & $4.20,4.80$ \\
\hline
\end{tabular}

$K 1$, flat keratometry; $K 2$, steep keratometry; $A C D$, anterior chamber depth; $A L$, axial length; $P C D$, programmed capsulorhexis diameter; SD, standard deviation; $y$, years

Anterior capsulorhexis was performed with femtosecond laser. No patient developed an anterior capsule tear. IOL implantation was successfully performed in all 28 cases. Tecnis ZCB00 (Abbott Medical Optics, 
United States), which is a hydrophobic acrylic 1-piece IOL with 6.0-mm optic diameter and 13.0-mm overall diameter, was implanted into the capsular bag. Posterior capsulotomy was successfully performed with anterior vitrectomy in all cases without any posterior capsule ruptures. Capsulorhexis enlargement occurred in all 28 cases (Figure 2).

Table 2 shows the PCD, AACD, and "E" for each patient. "E" was estimated to be $1.211 \pm 0.039$ (standard deviation [SD]; range, 1.122-1.284).

Table 2: Clinical data of patients. 


\begin{tabular}{|c|c|c|c|c|c|c|}
\hline \multirow[t]{2}{*}{ Patient } & \multirow[t]{2}{*}{ Case } & \multirow[t]{2}{*}{ Eye } & \multirow[t]{2}{*}{ Age at Surgery (y) } & \multicolumn{3}{|c|}{ Femtosecond Laser Capsulorhexis } \\
\hline & & & & PCD & AACD & $E$ \\
\hline Patient 1 & 1 & $\mathrm{~L}$ & 6.83 & 4.5 & 5.48 & 1.218 \\
\hline Patient 2 & 2 & $\mathrm{~L}$ & 4.50 & 4.5 & 5.47 & 1.216 \\
\hline Patient 3 & 3 & $\mathrm{R}$ & 6.00 & 4.6 & 5.57 & 1.211 \\
\hline Patient 4 & 4 & $\mathrm{~L}$ & 5.00 & 4.6 & 5.55 & 1.207 \\
\hline Patient 5 & 5 & $\mathrm{R}$ & 6.75 & 4.8 & 5.72 & 1.192 \\
\hline \multirow[t]{2}{*}{ Patient 6} & 6 & $\mathrm{R}$ & 3.50 & 4.6 & 5.64 & 1.226 \\
\hline & 7 & $\mathrm{~L}$ & 3.50 & 4.4 & 5.26 & 1.195 \\
\hline Patient 7 & 8 & $\mathrm{R}$ & 5.92 & 4.6 & 5.54 & 1.204 \\
\hline \multirow[t]{2}{*}{ Patient 8} & 9 & $\mathrm{R}$ & 6.83 & 4.6 & 5.61 & 1.220 \\
\hline & 10 & $\mathrm{~L}$ & 6.83 & 4.6 & 5.59 & 1.215 \\
\hline \multirow[t]{2}{*}{ Patient 9} & 11 & $\mathrm{R}$ & 3.25 & 4.6 & 5.67 & 1.233 \\
\hline & 12 & $\mathrm{~L}$ & 3.33 & 4.4 & 5.48 & 1.245 \\
\hline Patient 10 & 13 & $\mathrm{~L}$ & 4.08 & 4.4 & 5.20 & 1.182 \\
\hline \multirow[t]{2}{*}{ Patient 11} & 14 & $\mathrm{~L}$ & 3.00 & 4.4 & 5.47 & 1.243 \\
\hline & 15 & $\mathrm{R}$ & 3.00 & 4.4 & 5.46 & 1.241 \\
\hline Patient 12 & 16 & $\mathrm{~L}$ & 2.08 & 4.5 & 5.78 & 1.284 \\
\hline Patient 13 & 17 & $\mathrm{~L}$ & 6.92 & 4.5 & 5.45 & 1.211 \\
\hline Patient 14 & 18 & $\mathrm{R}$ & 4.83 & 4.4 & 5.17 & 1.175 \\
\hline Patient 15 & 19 & $\mathrm{R}$ & 5.50 & 4.4 & 5.14 & 1.168 \\
\hline Patient 16 & 20 & $\mathrm{R}$ & 2.75 & 4.4 & 5.45 & 1.239 \\
\hline \multirow[t]{2}{*}{ Patient 17} & 21 & $\mathrm{R}$ & 4.33 & 4.4 & 4.95 & 1.125 \\
\hline & 22 & $\mathrm{~L}$ & 4.58 & 4.4 & 5.02 & 1.141 \\
\hline Patient 18 & 23 & $\mathrm{~L}$ & 3.92 & 4.4 & 5.60 & 1.273 \\
\hline Patient 19 & 24 & $\mathrm{~L}$ & 6.33 & 4.5 & 5.05 & 1.122 \\
\hline Patient 20 & 25 & $\mathrm{~L}$ & 2.25 & 4.4 & 5.38 & 1.223 \\
\hline Patient 21 & 26 & $\mathrm{R}$ & 3.33 & 4.4 & 5.55 & 1.261 \\
\hline Patient 22 & 27 & $\mathrm{~L}$ & 5.75 & 4.2 & 5.10 & 1.214 \\
\hline
\end{tabular}


AACD, actual achieved capsulorhexis diameter; PCD, programmed capsulorhexis diameter; y, years; L, left; $\mathrm{R}$,

right; E, index of enlargement ( $\left.\frac{\text { actual achieved capsulorhexis diameter }}{\text { programmed capsulorhexis diam eter }}\right)$

\section{Correlations among the different parameters and " $E$ "}

Simple correlation analysis showed " $E$ " was negatively related to the age at surgery $(r=-0.417, p=0.027)$ (Figure 3) and ACD ( $r=-0.558, p=0.002)$ (Figure 4).

Results of partial correlation analysis showed that " $E$ " value correlated significantly with ACD $(r=-0.469$, $p=0.021)$ and $A L(r=0.452, p=0.027)$, but showed only a weak correlation with the age at surgery $(r=-0.343, p=0.100), K 1(r=-0.253, p=0.232)$ and $K 2(r=0.072, p=0.737)$.

\section{Multiple Linear Regression}

Multiple linear regression was performed using the age at surgery, AL, ACD, K1 and K2; the results showed that "E" correlated positively with AL, whereas it correlated negatively with ACD (Table 3).

Table 3. Multiple linear regression for "E."

\begin{tabular}{|llll|}
\hline Variables & Beta & SE & $p$ value \\
ACD & -0.052 & 0.014 & 0.001 \\
AL & 0.009 & 0.004 & 0.046 \\
Constant & 1.177 & 0.038 & $<0.001$ \\
\hline
\end{tabular}

PCD, programmed capsulorhexis diameter; ACD, anterior chamber depth; AL, axial length; SE, standard error;

The multiple linear regression model was based on the following equation: Predicted $E=$ 1.177-0.052 $\times A C D+0.009 \times A L, \mathrm{R}^{2}=0.346(\mathrm{~F}=4.396, \mathrm{p}=0.046)$. Furthermore, the predicted $\mathrm{PCD}$ formula (Equation 1 ) was obtained from the "predicted $\mathrm{E}^{\text {" equation }}$

$$
\text { Predicted } P C D=\frac{\text { attempted capsulorheis diameter }}{1.177-0.052 \times A C D+0.009 \times A L}
$$




\section{Discussion}

The age of primary IOL implantation in pediatric cataract surgery is an on-going debate, with the general consensus being 2 years old, and the feasibility of IOL implantation in children under 2 years old remains debatable ${ }^{4,10,11}$. In this study, 2-6-year-old children were included. To minimize the effects of some other surgical factors on anterior capsulorhexis, all 28 cases underwent the same surgical procedure: FLACS with primary $\mathrm{IOL}$ implantation and posterior capsulotomy with anterior vitrectomy. $\mathrm{IOL}$ is considered as the reference object to measure the AACD. All the patients underwent implantation with the Tecnis ZCB00 IOLs. The ACD, AL, and IOL power of 6 cases were measured with A-scan ultrasound in children who could not cooperate with the IOL-Master examination by the same experienced technician ${ }^{12}$. Posterior capsule capsulotomy was successfully performed in all cases to avoid PCO because young children may not comply with the Nd:YAG laser capsulotomy.

From 2009, femtosecond laser technology, which uses optical coherence tomography to perform realtime imaging of the anterior segment, has been successfully integrated into cataract surgery ${ }^{13,14}$. Previous studies have reported that femtosecond laser-assisted CCC has excellent advantages in adults such as more precise, accurate, reproducible, and predictable outcomes ${ }^{15,16}$. However, for children, high capsule elasticity generally results in an immediate significant increase in the capsulorhexis diameter after laser capsulorhexis ${ }^{17-19}$. In this study, anterior capsulorhexis enlargement occurred in all cases. Currently, when selecting the optimal PCD before femtosecond laser treatment, surgeons only can make judgments based on personal empirical rules. There is no clinically recommended value that can be used to avoid oversized opening of the anterior capsules; therefore, the actual achieved capsulotomy diameter is not always perfect.

A prospective study of 22 pediatric eyes (aged, $0.17-18$ years) have reported that the age at surgery was the most important factor which influenced capsulorhexis enlargement, with younger children having a greater increase in the actual capsulotomy diameter than older children ${ }^{20}$. Figure 3 shows the negative correlation of " $E$ " value with the age at surgery. This result is consistent with the former research. But partial correlation analysis and multiple linear regression analysis have showed no significant correlation of " $E$ " value with the age at surgery. Maybe it's because we have narrowed down the age group. In the 26-year-old age group, the influence of age on the degree of capsulorhexis enlargement may not be considered. More parameters were included in this study. Figure 4 shows the negative correlation of " $E$ " value with ACD. For the same PCD, a shallow preoperative ACD led to a large size of actual capsulorhexis diameter. ACD in the 28 cases examined in this study ranged from $1.57-3.93 \mathrm{~mm}$. Of note, the intracapsular pressure and the anterior capsule tension may be higher in the eyes with shallower preoperative ACD. Moreover, the possible laxity of the lens zonular fibers may lead to greater changes in shallower ACD before and after surgery. A combination of the two factors maybe lead to more obvious enlargement of the capsulorhexis size after the femtosecond laser treatment. The AL of the 28 cases ranged from 20.14-27.82 $\mathrm{mm}$. Data in table 3 indicates that " $E$ " correlated positively with AL. The authors speculate it may be due to the laxity of the lens zonular fibers also. However, the exact 
mechanism needs further study. Results of multiple regression analysis showed that " $E$ " is closely related to $A L$ and $A C D$. The predicted PCD formula (Equation 1) is obtained based on the multiple linear equation of "Predicted E."

The ideal CCC diameter should be slightly smaller than the optic diameter of the $\mathrm{IOL}^{21}$. The mean " $\mathrm{E}$ " value in this study was $1.211 \pm 0.039$ (SD) (range, 1.122-1.284). In clinical settings, according to the attempted capsulorhexis diameter, a PCD can be roughly calculated using the "E" value. Meanwhile, the predicted formula can provide clinicians with more accurate PCD values before the femtosecond laser procedure.

This study is limited by a small sample size. A larger sample size might show a more accurate consequence of prediction. To make the predicted PCD calculations convenient and faster, the following study will propose development of an automatic and intelligent software platform on which doctors only need to input $A C D, A L$, and attempted capsulorhexis diameter, and then the PCD could be generated immediately.

\section{Conclusion}

The achieved capsulorhexis size is significantly larger than the programmed value for children after femtosecond laser-assisted anterior capsulorhexis. For the 2-6-year-old patients with congenital cataract, the "E" value and the predicted formula related to AL and ACD may help to set an optimal PCD to obtain a more accurate achieved capsulorhexis size.

\section{Abbreviations}

CCC: continuous circular capsulorhexis; IOL: intraocular lens; FLACS: femtosecond laser-assisted cataract surgery; $E$ : index of enlargement; AACD: actual achieved capsulorhexis diameter; PCD: programmed capsulorhexis diameter; $\mathrm{ACD}$ : anterior chamber depth; $\mathrm{AL}$ : axial length; $\mathrm{K} 1$ : flat keratometry; $\mathrm{K} 2$ : steep keratometry

\section{Declarations}

\section{Ethics approval and consent to participate:}

Ethical committee approval was sought from the Central South University and Changsha Aier Eye Hospital Review Board. The study was conducted adhering to the declaration of Helsinki involving human participants and the approved guidelines. After introducing ophthalmologic examination and surgical methods to patients' guardians and parents, written informed consent of the measurement data, surgical video recording and publication of images and findings in this study was obtained from them.

\section{Consent for publication:}


Written informed consent was obtained from the patients' guardians and parents for publication of the data and any accompanying images. A copy of the written consent is available for review by the Editor of this journal.

\section{Availability of data and materials:}

The datasets used and/or analyzed during the current study are available from the corresponding author on reasonable request.

\section{Competing interests:}

The authors declare that there are no conflicts of interest regarding the research, authorship, and cpublication of this paper.

\section{Funding:}

The financial support for the conduct of the research was provided by the Changsha Science and Technology Bureau (KQ1706015), Hunan, China. The funder had no involvement in the design of the study and collection, analysis, and interpretation of data and in writing the manuscript.

\section{Authors' contributions:}

ML L, QY T and D L designed research; $M L L, D$ and $S L$ conducted research; $M L L$ and WW $Z$ performed statistical analysis; ML L and QY T wrote the draft of the manuscript. All authors read, reviewed and approved the final manuscript.

\section{Acknowledgements:}

Not applicable

\section{References}

1. Wu X, Long E, Lin H, Liu Y. Prevalence and epidemiological characteristics of congenital cataract: A systematic review and meta-analysis. Sci Rep 2016; 6(1): 28564

2. Wilson ME, Pandey SK, Thakur J. Paediatric cataract blindness in the developing world: Surgical techniques and intraocular lenses in the new millennium. Brit J Ophthalmol 2003; 87(1): 14-19

3. Hensch TK, Quinlan EM. Critical periods in amblyopia. Visual Neurosci 2018; 35: E14

4. Repka MX, Dean TW, Kraker RT, Bothun ED, Morrison DG, Lambert SR, et al. Visual acuity and ophthalmic outcomes in the year after cataract surgery among children younger than 13 years. Jama Ophthalmol 2019; 137(7): 817-824

5. Bang SP, Yoo Y, Jun JH, Joo C. Effects of Residual Anterior Lens Epithelial Cell Removal on Axial Position of Intraocular Lens after Cataract Surgery. J Ophthalmol 2018; 2018:9704892. 
6. Findl O, Hirnschall N, DraschI P, Wiesinger JO. Effect of manual capsulorhexis size and position on intraocular lens tilt, centration, and axial position. J Cataract Refr Surg 2017; 43(7): 902-908

7. Li S, Hu Y, Guo R, Shao Y, Zhao J, Zhang J, et al. The effects of different shapes of capsulorrhexis on postoperative refractive outcomes and the effective position of the intraocular lens in cataract surgery. BMC Ophthalmol 2019; 19(1): 59

8. Mastropasqua L, Toto L, Mattei PA, Vecchiarino L, Mastropasqua A, Navarra R, et al. Optical coherence tomography and 3-dimensional confocal structured imaging system-guided femtosecond laser capsulotomy versus manual continuous curvilinear capsulorhexis. J Cataract Refr Surg 2014; 40(12): 2035-2043

9. Chen X, Xiao W, Ye S, Chen W, Liu Y. Efficacy and safety of femtosecond laser-assisted cataract surgery versus conventional phacoemulsification for cataract: A meta-analysis of randomized controlled trials. Sci Rep 2015; 5: 13123

10. Lim ME, Buckley EG, Prakalapakorn SG. Update on congenital cataract surgery management. Curr Opin Ophthalmol 2017; 28(1): 87-92

11. Solebo AL, Russell-Eggitt I, Cumberland PM, Rahi JS. Risks and outcomes associated with primary intraocular lens implantation in children under 2 years of age: The loLunder2 cohort study. $\mathrm{The} \mathrm{Br} \mathrm{J}$ Ophthalmol 2015; 99(11): 1471-1476

12. Grulkowski I, Liu JJ, Zhang JY, Potsaid B, Jayaraman V, Cable AE, et al. Reproducibility of a longrange swept-source optical coherence tomography ocular biometry system and comparison with clinical biometers. Ophthalmology 2013; 120(11): 2184-2190

13. Palanker DV, Blumenkranz MS, Andersen D, Wiltberger M, Marcellino G, Gooding P, et al. Femtosecond laser-assisted cataract surgery with integrated optical coherence tomography. Sci Transl Med 2010; 2(58): 58r-85r

14. Nagy Z, Takacs A, Filkorn T, Sarayba M. Initial clinical evaluation of an intraocular femtosecond laser in cataract surgery. J Refract Surg 2009; 25(12): 1053-1060

15. Schultz T, Joachim SC, Tischoff I, Dick HB. Histologic evaluation of in vivo femtosecond lasergenerated capsulotomies reveals a potential cause for radial capsular tears. Eur J Ophthalmol 2015; 25(2): 112-118

16. Alió JL, Abdou AA, Puente AA, Zato MA, Nagy Z. Femtosecond Laser Cataract Surgery: Updates on Technologies and Outcomes. J Refract Surg 2014; 30(6): 420-427

17. Andreo LK, Wilson ME, Apple DJ. Elastic properties and scanning electron microscopic appearance of manual continuous curvilinear capsulorhexis and vitrectorhexis in an animal model of pediatric cataract. J Cataract Refr Surg 1999; 25(4): 534-539

18. Guo S, Wagner RS, Caputo A. Management of the anterior and posterior lens capsules and vitreous in pediatric cataract surgery. J Pediatr Ophthalmol Strabismus 2004; 41(6): 330-337

19. Dick HB, Schultz T. Femtosecond laser-assisted cataract surgery in infants. J Cataract Refr Surg 2013; 39(5): 665-668 
20. Dick HB, Schelenz D, Schultz T. Femtosecond laser-assisted pediatric cataract surgery: Bochum formula. J Cataract Refr Surg 2015; 41(4): 821-826

21. Szigeti A, Kránitz K, Takacs Al, Miháltz K, Knorz MC, Nagy ZZ. Comparison of long-term visual outcome and IOL position with a single-optic accommodating IOL After 5.5- or 6.0-mm Femtosecond laser capsulotomy. J Refract Surg 2012; 28(9): 609

\section{Figures}

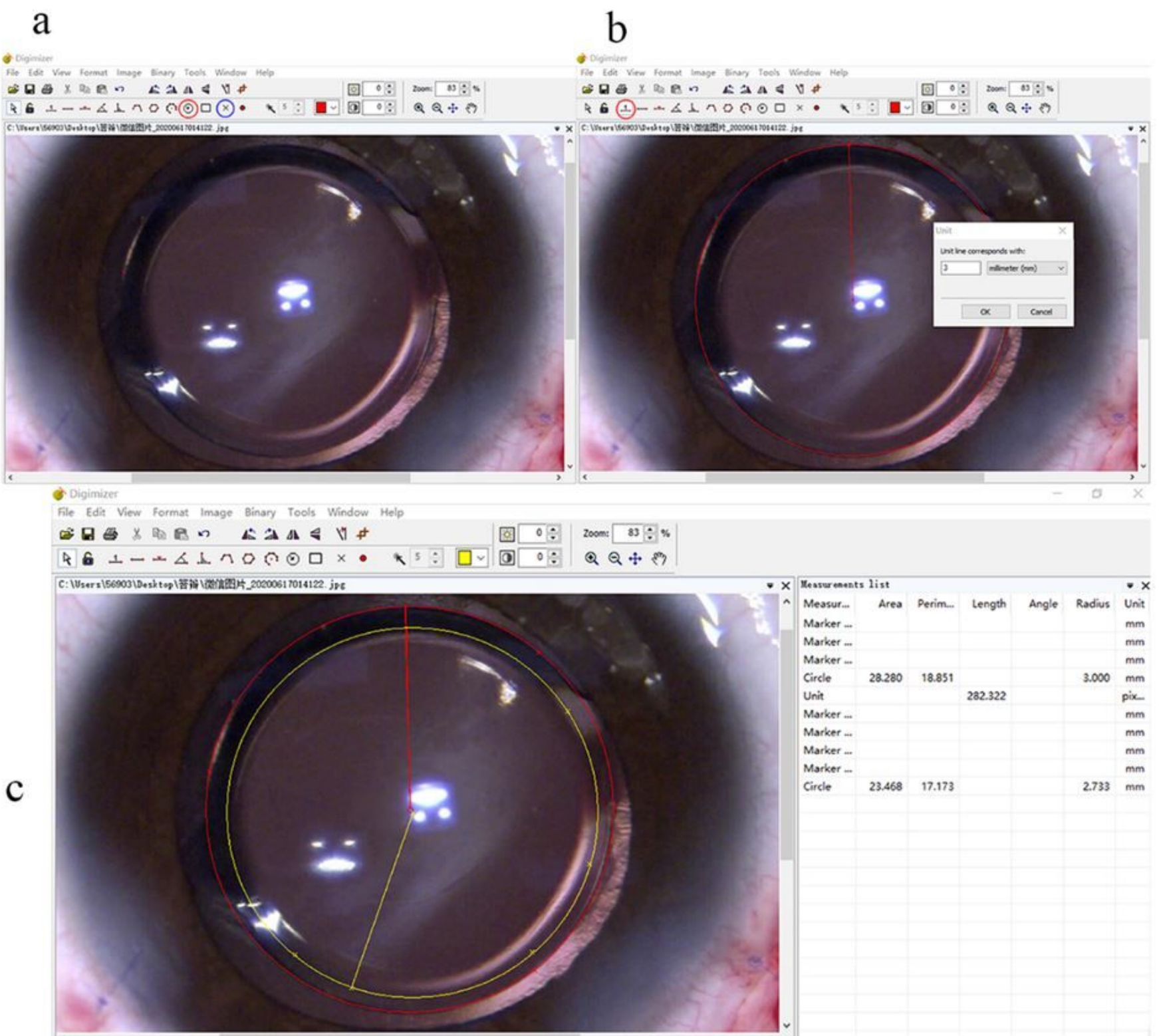

\section{Figure 1}

Measurement of the actual achieved capsulorhexis. a: Select three points using the "marker style 1" (circled in blue). Delineate the boundary of the IOL optic using the "circle to center" (circled in red) to get the radius of IOL optic. b: Click on the "Unit" button (circled in red). Use the radius of IOL optic to 
determine the unit. For example, if the diameter of IOL optic is $6.0 \mathrm{~mm}$, type " $3 \mathrm{~mm}$ " in the unit input box. c: Delineate the boundary of the actual achieved capsulorhexis using the "circle to center" button to acquire the radius of capsulorhexis from the "measurement list" on the right side of the screen.

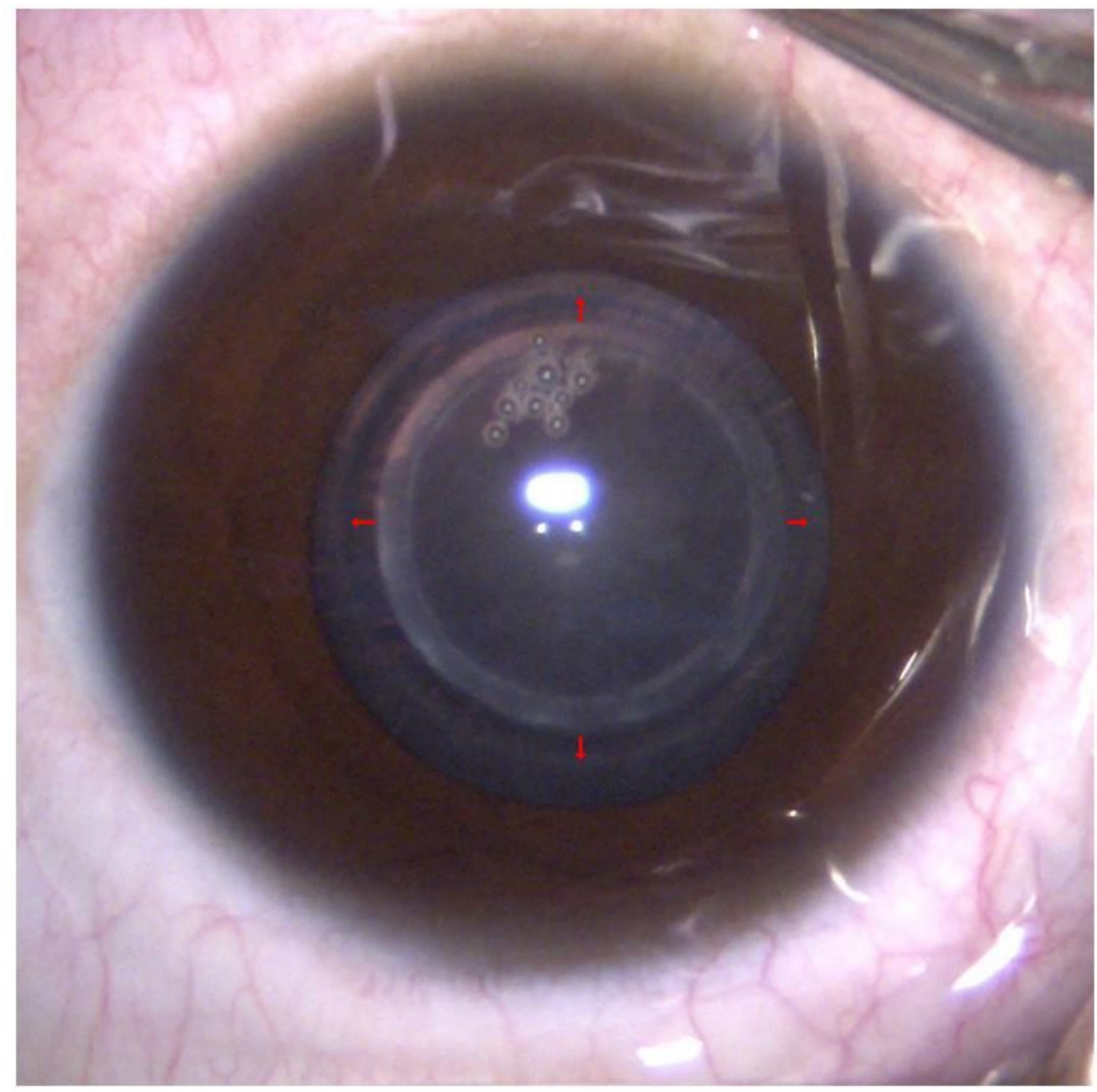

\section{Figure 2}

Symmetrical capsulorhexis enlargement immediately after femtosecond laser-assisted capsulorhexis in a child with congenital cataract. 


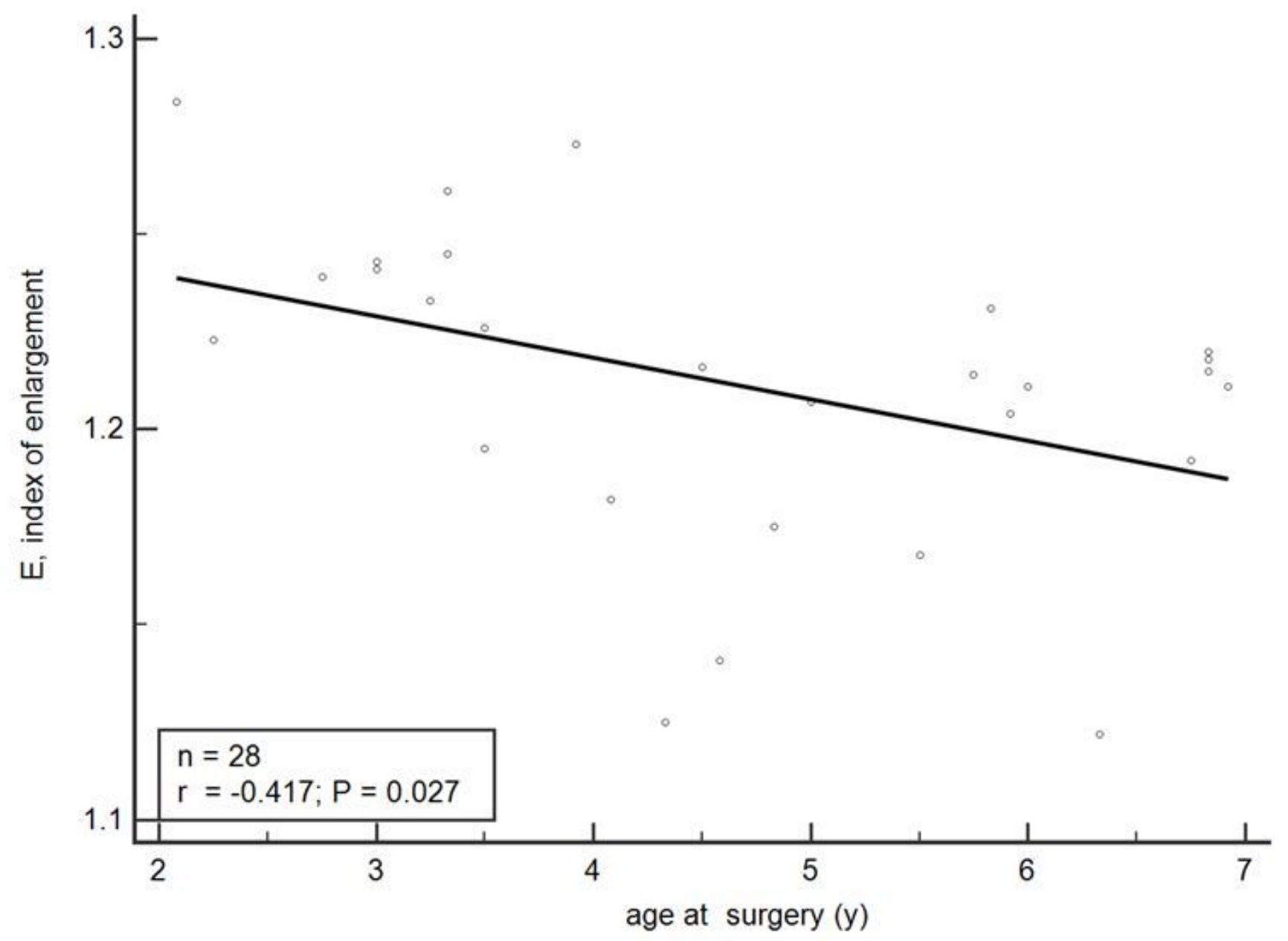

Figure 3

Relationship between " $E$ " and the age at surgery. E, index of enlargement. 


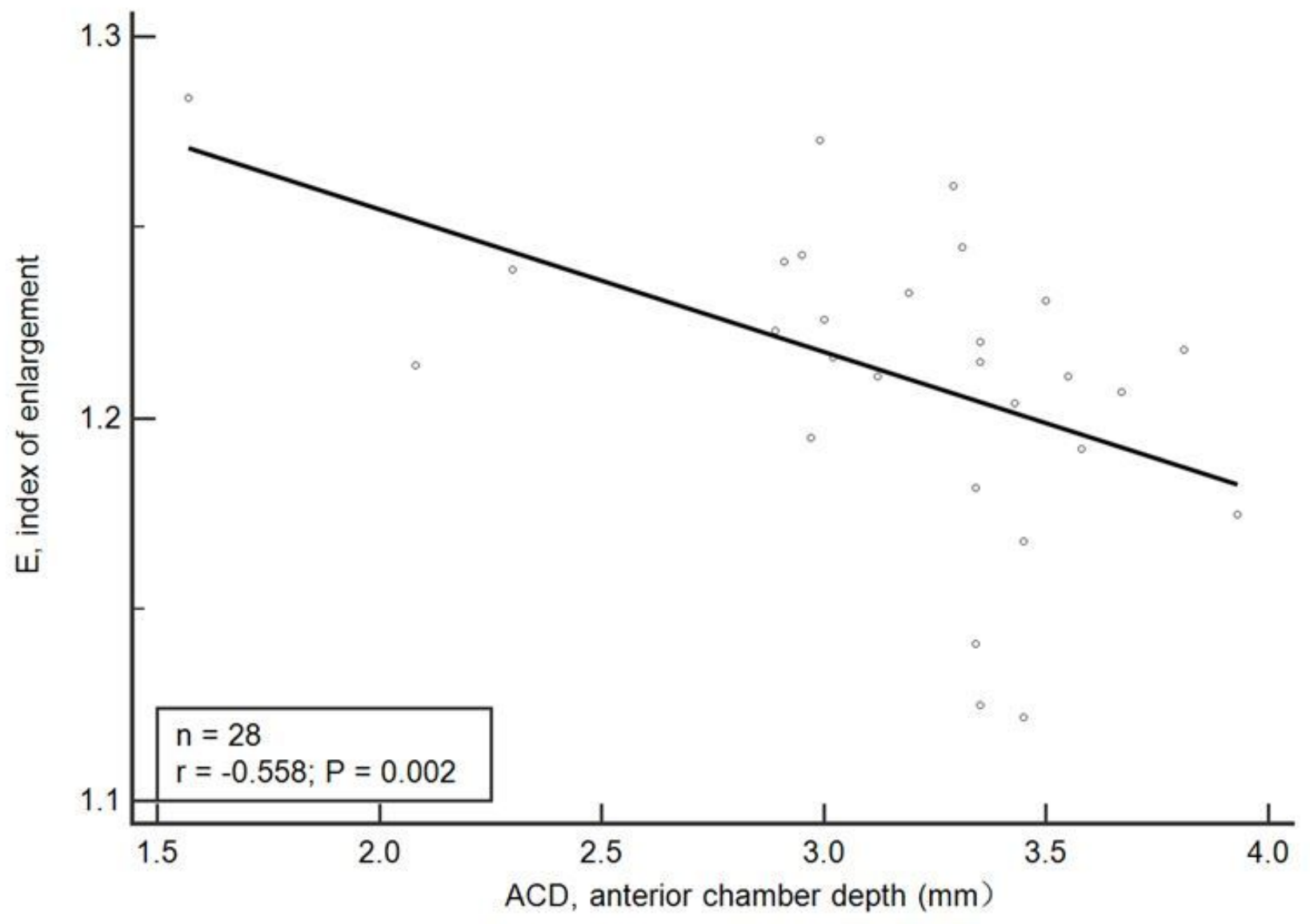

Figure 4

Relationship between " $E$ " and ACD. E, index of enlargement; $A C D$, anterior chamber depth. 